\title{
Functional Analysis of a Tannic-Acid-Inducible and Hypoviral-Regulated Small Heat-Shock Protein Hsp24 from the Chestnut Blight Fungus Cryphonectria parasitica
}

\author{
Jin-Ho Baek, ${ }^{1}$ Jin-Ah Park, ${ }^{1}$ Jung-Mi Kim, ${ }^{2}$ Jung-Mi Oh, ${ }^{1}$ Seung-Moon Park, ${ }^{1}$ and Dae-Hyuk Kim ${ }^{1}$ \\ ${ }^{1}$ Institute for Molecular Biology and Genetics, Center for Fungal Pathogenesis, Chonbuk National University, Jeonju, Chonbuk \\ 561-756, Korea; ${ }^{2}$ Department of Bio-Environmental Chemistry, Wonkwang University, Iksan, Chonbuk 570-749, Korea
}

Submitted 23 June 2013. Accepted 1 September 2013.

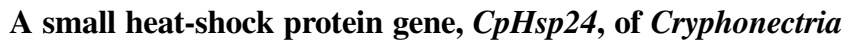
parasitica was selected based on its expression pattern, which showed that it was tannic acid inducible and that its induction was severely hampered by a hypovirus. The predicted protein sequence of $\mathrm{CpHsp24}$ consisted of a hallmark $\alpha$-crystalline domain flanked by a variable $\mathrm{N}$-terminal and a short C-terminal region. Disruption of $\mathrm{CpHsp24}$ resulted in a slow growth rate under standard growth conditions. The CpHsp24-null mutant showed enhanced sensitivity to heat shock, which was consistent with Northern and Western analyses displaying the heat-shock induction of the $\mathrm{CpHsp24}$ gene and protein, respectively. Virulence tests on the excised bark revealed a severe decrease in the necrotic area of the CpHsp24-null mutant. When the hypovirus was transferred, virus-containing $C p H s p 24-n u l l$ progeny displayed severely retarded growth patterns with hypovirulent characteristics of reduced pigmentation and sporulation. Because the tannic-acid-inducible and hypoviralsuppressible expression and the severely impaired virulence are also characteristics of the laccase 3 gene (lac3), lac3 expression in the CpHsp24-null mutant was also examined. The resulting lac 3 induction was severely affected in the CpHsp24-null mutant, suggesting that $\mathrm{CpHsp24}$ is important for lac3 induction and that $\mathrm{CpHsp24}$ may act as a molecular chaperone for the lac3 protein.
\end{abstract}

Cryphonectria parasitica (Murrill) M. E. Barr, the causal agent of chestnut blight, has devastated both European and American chestnut forests in the first part of the 20th century (Van Alfen 1982). A recent survey in Korea, where chestnut blight was believed to cause only mild problems, indicated that disease severity has become more pronounced; more than $30 \%$ of necrotic lesions of chestnut trees in Korea were infected with C. parasitica (Ju et al. 2002) and variations in disease severity were observed among currently available chestnut varieties that were previously considered to be disease-resistant (Lee et al. 1999). However, fungal strains infected with a singlestranded (ss)RNA virus, Cryphonectria hypovirus 1 (CHV1), exhibited the characteristic symptoms of lowered virulence, a

Corresponding author: D.-H. Kim; Telephone: +82-63-270-3440; Fax: +82-63-270-4312; E-mail: dhkim@ chonbuk.ac.kr

* The $\boldsymbol{e}$-Xtra logo stands for "electronic extra" and indicates that two supplementary figures are published online.

(C) 2014 The American Phytopathological Society phenomenon referred to as hypovirulence (Anagnostakis 1982; Nuss 1992; Van Alfen et al. 1975), with diverse hypovirulenceassociated phenotypic changes such as reduced sporulation, pigmentation, female fertility, laccase production, and oxalate accumulation (Elliston 1985; Havir and Anagnostakis 1983; Rigling et al. 1989). The molecular basis for these phenotypic changes was attributed to the hypoviral modulations in host transcriptional and translational profiles (Allen and Nuss 2004; Allen et al. 2003; Deng et al. 2007; Kang et al. 1999; Kazmierczak et al. 1996, 2012; Kim et al. 2012; Wang et al. 2013).

Systemic analysis of transcriptional and translational changes regarding the virulence of forest fungal pathogens, such as chestnut blight fungus, to their native hosts has been considered to be more difficult than the analysis of grass pathogens (Chung et al. 2008). However, supplementation with tannic acid (TA), which is abundant in the bark of chestnut trees and is assumed to be one of the major barriers against pathogen infection, is believed to be successful in imitating wood induction in $C$. parasitica and to address some, not all, of the fungal changes that occur in its native host for aspects of pathogenesis and fungal gene regulation by the hypovirus (Chung et al. 2008; Rigling et al. 1989). Moreover, a recent study demonstrated the feasibility of the proteomic analysis of $C$. parasitica grown in the presence of TA, which is known to precipitate protein products (Kim et al. 2012). Therefore, the systemic analysis of transcriptional and translational products in the presence of TA has been established and is expected to foster in-depth understanding of plant-fungus-mycovirus interactions.

Among the several fungal regulatory pathways perturbed by hypovirus infection, the stress-response pathway has been of interest because of its specificity in hypoviral perturbation (Allen and Nuss 2004; Allen et al. 2003). However, few studies have been conducted on the biological function of the components of the stress-response pathway in this fungus (Lim et al. 2010).

Heat-shock response is a molecular reaction to stressful but sublethal temperature and is characteristic of all living organisms, including bacteria, fungi, plants, and animals (Lindquist and Craig 1988; Plesofsky 2004). During the initial stage of the heat-shock response, normal activities of transcription and translation are drastically reduced and massive quantities of heat-shock proteins (Hsp) are produced. Hsp, a group of molecular chaperones found in all organisms, accumulate upon shifts from a physiological temperature to higher temperatures. These proteins are also induced by other stresses, allowing cells to adapt and survive under challenging conditions. The heat-shock response is ubiquitous and prominent Hsp are

56 / Molecular Plant-Microbe Interactions 
highly conserved among all groups of organisms. Many Hsp are essential proteins that contribute to cellular metabolism during normal growth but are strongly induced during specific stages of organelle biogenesis, protein folding and transport, transcriptional activation mechanisms, stress resistance, and differentiation (Lindquist and Craig 1988; Plesofsky 2004).

According to their molecular weight, Hsp have been classified into several classes or families, and a cell may express multiple members of the same family (Walter and Buchner 2002). There are five major families of Hsp (Buchner 1996; Richter et al. 2010); four of them-Hsp100s, Hsp90s, Hsp70s, and Hsp60s - consist of ATP-dependent high molecular mass Hsp, while the fifth family - the small Hsp (sHsp) - consists of ATP-independent low molecular mass Hsp with sizes of 12 to $42 \mathrm{kDa}$ (Jaya et al. 2009) and a conserved sequence in their C-terminus called the $\alpha$-crystalline domain (de Jong et al. 1993; Garstel et al. 1997; Narberhaus 2002). Unlike other families of Hsp, sHsp show extensive sequence variation and evolutionary divergence. However, compared with the high molecular mass Hsp, fewer sHsp have been identified and functional studies of sHsp are considerably limited.

In this study, we report the characterization of a TA-inducible and hypoviral-regulated sHsp from $C$. parasitica. By construction of the corresponding null mutant, we demonstrated the biological function of the cloned sHsp and suggested how the protein product of the cloned $s H s p$ gene might be involved in the virulence of this fungus.

\section{RESULTS}

Cloning and characterization of a TA-responsive and hypoviral-regulated $C$. parasitica Hsp 24 gene.

RNA differential display using the GeneFishing method, a polymerase chain reaction (PCR)-based differential display technique, resulted in more than 30 PCR amplicons showing the expression pattern in response to TA and hypovirus infection. Among them, a 401-bp PCR amplicon exhibiting an expression pattern of induction by TA that was alleviated by hypovirus infection was selected and further characterized. Northern blot analysis was conducted to verify the PCR-based expression pattern. The accumulation of transcript in virus-free EP155/2 was highly induced by the TA supplement (Fig. 1). However, its induction pattern was significantly decreased in virus-infected UEP1. These Northern blot analyses of the hypovirus-regulated TA-induced pattern were in a good agreement with the findings from the GeneFishing technique.

Sequence analysis of the cloned PCR amplicon revealed a high homology with known sHsp genes. The highest sHsp homolog was the sHsp30 of Metarhizium acridum, which shared $37 \%$ identity at the amino acid level. By inspection of the draft genome sequence and sequence comparison with other known sHsp, a 4,047-bp fragment, which was expected to contain a full-length $s H s p$ gene, was amplified using genomic PCR. Southern blot analysis of restriction enzymedigested $C$. parasitica genomic DNA indicated that the cloned PCR amplicon was present as a single-copy gene in the $C$. parasitica genome (data not shown). In addition, based on the genomic sequence analysis, a near full-length cDNA clone was obtained using reverse-transcription (RT)-PCR with the primer pair $C$. parasitica Hsp 24 ( $\mathrm{CpHsp} 24)-\mathrm{cF} 1$

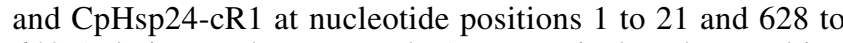
648 (relative to the start codon), respectively. The resulting 648-bp amplicon was cloned into the pGEM-T Easy Vector (Promega Corp., Madison, WI, U.S.A.). A sequence comparison with the corresponding genomic sequence revealed that the cloned gene appeared to be intron-less, which is common in other fungal sHsp.
Sequence analysis in the promoter region of the CpHsp24 gene revealed canonical CAAT and TATAA boxes at -191 and -149 nucleotides (nt), respectively (Supplementary Fig. S1A). The minimum consensus sequence of heat-shock element nGAAnnTTCnnGAAn (Tachibana et al. 2002) was not found up to $5.0 \mathrm{~kb}$ upstream of the start codon. However, within 2.0 $\mathrm{kb}$, there appeared to be four nonconventional heat-shock response element (HSE) with a 5-bp insertion between two repeating pentameric units, nGAAn-(5-bp)-nGAAn, at -94, $-1,378,-1,738$, and $-1,876 \mathrm{nt}$ from the start codon (Tachibana et al. 2002). In addition, analysis of the promoter region revealed the presence of two stress response element (STRE) motifs (core sequence AGGGG at -1,896 and CCCCT at $-1,406)$, a DNA element present in many stress-induced genes (Martinez-Pastor et al. 1996). The sequence around the first ATG was in good agreement with Kozak's consensus sequence in that the nucleotide -3 position was the A in AAAGATG.

The deduced protein product of the cloned gene consisted of 215 amino acids (aa), with an estimated molecular mass of $24.4 \mathrm{kDa}$ and an isoelectric point of 6.52 (the GenBank accession number for CpHsp24 is KF201573). Analysis of the deduced amino acid sequence revealed the presence of the conserved hallmark domain of sHsp, an $\alpha$-crystalline domain between 73 and 195 aa, which was flanked by a variable hydrophobic N-terminal region and a short $\mathrm{C}$-terminal extension. Homology searches using the deduced amino acid sequence indicated that the protein product of the cloned gene is related to the fungal sHsp from Neosartorya fischeri (35\%), Talaromyces marneffei (35\%), Aspergillus niger (33\%), Magnaporthe grisea $(30 \%)$, Neurospora crassa $(26 \%)$, Trichoderma reesei (26\%), and Saccharomyces cerevisiae (24\%). Multiple alignment of closely related sHsp revealed that the cloned gene maintained the conserved amino acids in the $\alpha$-crystalline domain
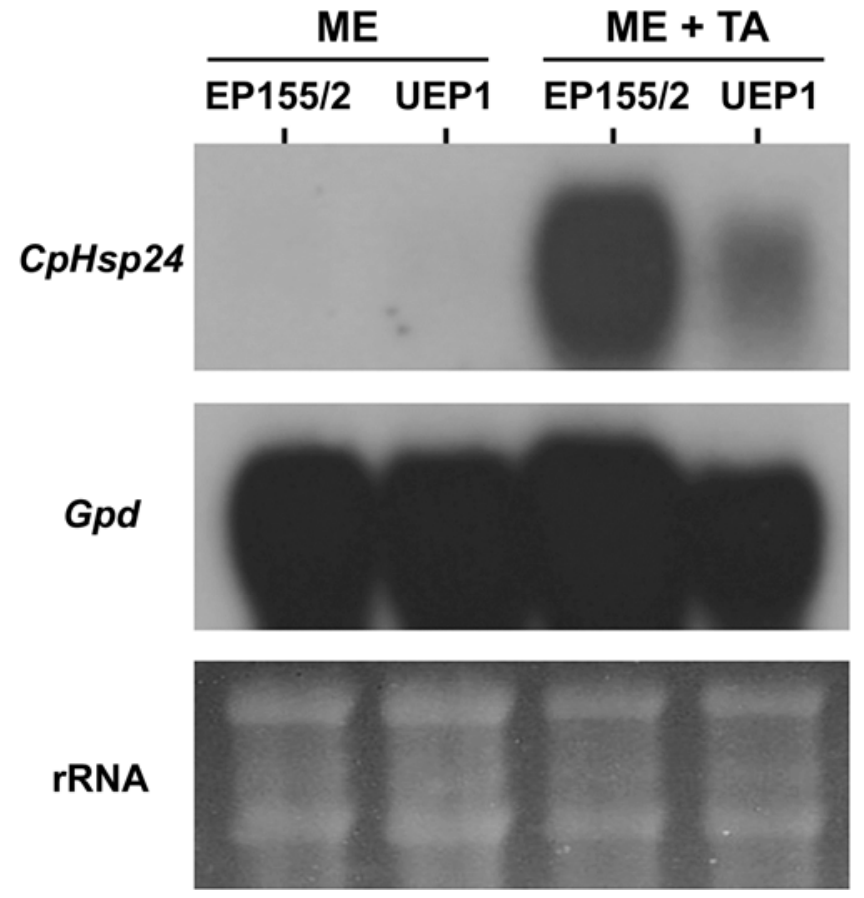

Fig. 1. Northern blot analysis of $C p H s p 24$ in response to tannic acid (TA) supplementation. Total RNA was extracted from the virus-free wild-type (EP155/2) and its isogenic virus-containing hypovirulent strain (UEP1). The identification of the strains is shown at the top of the lanes. ME and ME+TA, indicated above the panel, represent the absence and presence of TA supplementation, respectively. Equal loading of RNA samples was demonstrated in the bottom panels by a parallel blot hybridized with a glyceraldehyde-3-phosphate dehydrogenase $(G p d)$ probe as an internal control and the ethidium bromide-stained gel (rRNA). 
and there were two 6-aa extensions at the variable N-terminal region. In addition, the resulting phylogram indicated that the protein product of the cloned gene fell into a different cluster from the other containing those from $N$. crassa, T. reesei, $M$. grisea, Talaromyces marneffei, Neosartorya fischeri, and A. niger, with a high bootstrap value suggesting a genuine evolutionary relationship (Henikoff and Henikoff 1992). Together with the presence of the hallmark sequence of the $\alpha$-crystalline domain within the amino acid sequence, the significant homology to the known fungal sHsp, and the predicted molecular mass of the cloned gene, we referred to the cloned gene as CpHsp24.

\section{Regulation of the expression of CpHsp24.}

Because the cloned gene appeared to encode the sHsp, we examined the expression pattern of the gene at the protein level under the heat-shock condition. Western blot analysis of the protein product (CpHSP24) of the CpHsp24 gene revealed the major antigen-antibody reacting band at $37 \mathrm{kDa}$ in sodium dodecyl sulfate polyacrylamide gel electrophoresis (SDSPAGE) gel. The absence of the corresponding band at $37 \mathrm{kDa}$ in the CpHsp24-null mutant indicated that the band at $37 \mathrm{kDa}$ was specific to the protein product of the CpHsp24 gene. The molecular weight deduced from the DNA sequence of the gene was $24.4 \mathrm{kDa}$ while the specific antigen-antibody reacting band ran at $37 \mathrm{kDa}$ in SDS-PAGE gels. Considering the fact that there is no conventional glycosylation site Ser/Thr-X-Asn

A EP155/2 UEP1

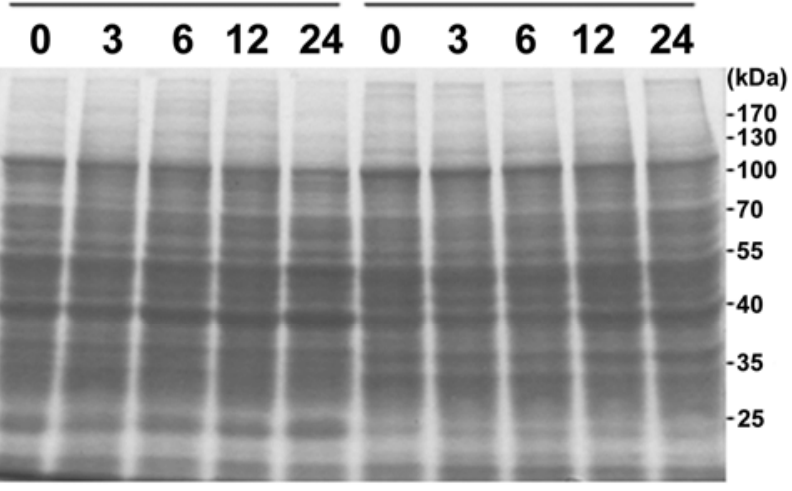

B

EP155/2
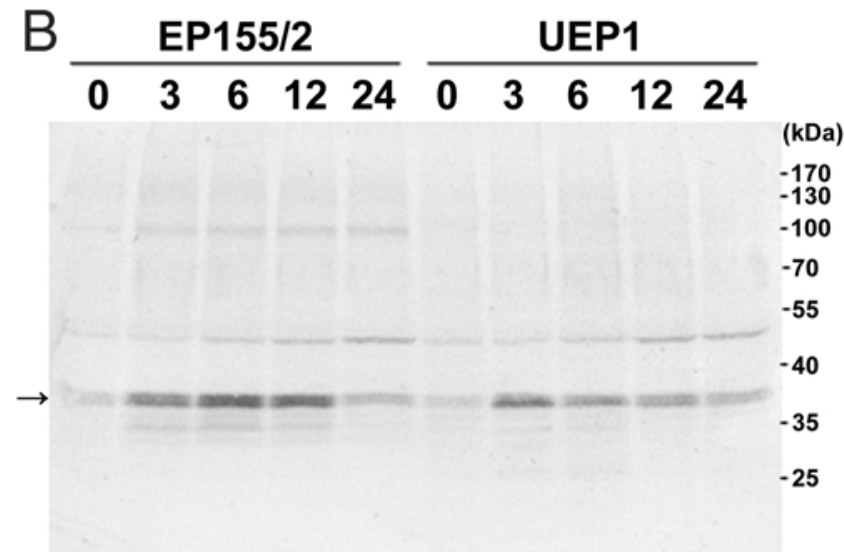

Fig. 2. Western blot analysis of CpHSP24 in response to heat shock conditions. A, Coomassie blue-stained sodium dodecyl sulfate polyacrylamide gel electrophoresis of cell-free protein extract of Cryphonectria parasitica. B, Antigen-antibody reaction of the corresponding gel. The arrowhead indicates the CpHSP24 protein. Strains designated above the line are the wild-type (EP155/2) and its isogenic hypovirulent strain (UEP1). Numbers at the top of the lanes refer to hours after heat shock. For each lane, $30 \mu \mathrm{g}$ of protein extract was loaded. in the deduced sequence of CpHSP24, the reason for these differences in molecular size estimates is currently unknown.

Western blot analysis indicated that the expression of CpHSP24 was induced, remained until $12 \mathrm{~h}$ after the heat shock, and then decreased at $24 \mathrm{~h}$ (Fig. 2). These induction patterns in response to heat-shock stress demonstrated that, indeed, the cloned gene encoded the Hsp. Interestingly, the induction pattern of CpHSP24, although not as distinctive as in TA induction, was hampered by the hypovirus, as suggested by the expression pattern of CpHSP24 from the hypovirus-infected UEP1 strain under the heat-shock condition.

\section{Construction of the CpHsp24-null mutant.}

To determine the biological function of the CpHsp24 gene, the CpHsp24-null mutant was constructed by site-directed recombination during integrative transformation. In total, 150 single-spored transformants with the disrupted $\mathrm{CpHsp24}$ gene designed for gene replacement were screened by PCR using the outer gene-specific and inner hygromycin phosphotransferase (hph) primers, which corresponded to $-1,360$ to $-1,340$ and 1,222 to 1,241 (relative to the start codon of CpHsp24 and hph, respectively). One transformant showed a PCR amplicon of the expected size, $2.9 \mathrm{~kb}$, corresponding to the disrupted alleles of the CpHsp24 gene. The putative CpHsp24-null mutant (TdHsp24-1) was further confirmed by Southern blot analysis (Supplementary Fig. S2). The hybridization pattern of SacIdigested genomic DNA of the three single-spored clones of CpHsp24-null mutant with the probe prepared using the 619-bp SacI-digested $\mathrm{CpHsp} 24$ fragment (probe A) differed from that of the wild type. In addition, the hybridization pattern of BamHIdigested genomic DNA of the three clones probed with the 1,350-bp BamHI-digested CpHsp24 fragment (probe B) differed from that of the wild type. These results suggested that the transforming vector had integrated at the CpHsp24 locus by sitedirected homologous recombination. Moreover, the SacI- and BamHI-digested genomic DNA of three clones of CpHsp24-null mutant had the hybridizing bands at 4.8 and $1.2 \mathrm{~kb}$, respectively, corresponding to the expected size of the replaced allele. These hybridizing patterns along with PCR confirmation indicated that
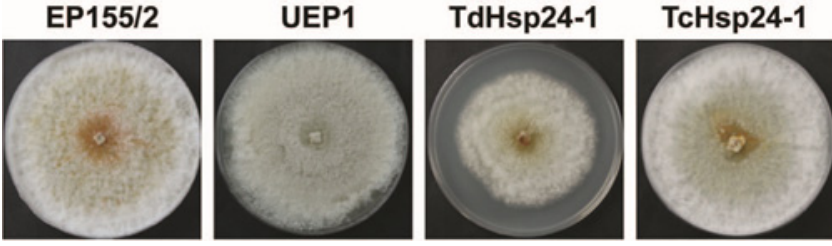

Fig. 3. Colony morphology of the CpHsp24-null mutant strain. Colonies after 10 days of culturing on potato dextrose agar supplemented with methionine and biotin are shown. Strains used, indicated above the panel, are the wild-type EP155/2, hypovirulent UEP1, CpHsp24-null mutant (TdHsp24-1), and the CpHsp24-complemented (TcHsp24-1) strains.

Table 1. Characteristics of null mutants compared with the wild type and hypovirulent strains ${ }^{\mathrm{a}}$

\begin{tabular}{lll}
\hline Strains & \multicolumn{1}{c}{ Characteristic } & \multicolumn{1}{c}{$\begin{array}{c}\text { Number of } \\
\text { conidia/ml }^{\mathbf{b}}\end{array}$} \\
\hline EP155/2 & Wild-type & $3.8 \times 10^{8} \pm 2.3 \times 10^{8}$ \\
TdHsp24-1 & CpHsp24-null mutant & $3.1 \times 10^{8} \pm 2.5 \times 10^{8}$ \\
TcHsp24-1 & CpHsp24-complemented strain & $3.5 \times 10^{8} \pm 2.7 \times 10^{8}$ \\
UEP1 & Hypovirulent & $2.5 \times 10^{6} \pm 1.1 \times 10^{6}$ \\
\hline
\end{tabular}

${ }^{a}$ Culture conditions were described previously (Kim et al. 1995).

${ }^{\mathrm{b}}$ Conidia from each plate were harvested with $10 \mathrm{ml}$ of sterile water and the number of conidia per plate was determined using a hemacytometer. Four replicates of each strain were used and the experiment was repeated three times. Values are means \pm standard deviation. 
the $C p H s p 24$ gene was replaced with part of the transforming vector rather than just disrupted, as was expected.

Phenotypic characteristics of the CpHsp24-null mutant.

The CpHsp24-null mutant had a retarded growth rate, as measured by the diameter of colonies on potato dextrose agar (PDA) supplemented with methionine and biotin (PDAmb), with less than $80 \%$ of the typical growth rate observed (Fig. 3). Pigmentation of the interwoven hyphal mat was observed as the wild-type EP155/2 (Fig. 3) and no difference in the conidiogenesis was observed (Table 1).

Because the heat-shock response of the cloned CpHsp24 gene was confirmed, we measured the sensitivity of the CpHsp24-null mutant to different temperatures (Fig. 4). When the CpHsp24 mutant was exposed to the heat-shock conditions, it showed further defects in its growth rate. Interestingly, the CpHsp24-null mutant exhibited more severely retarded
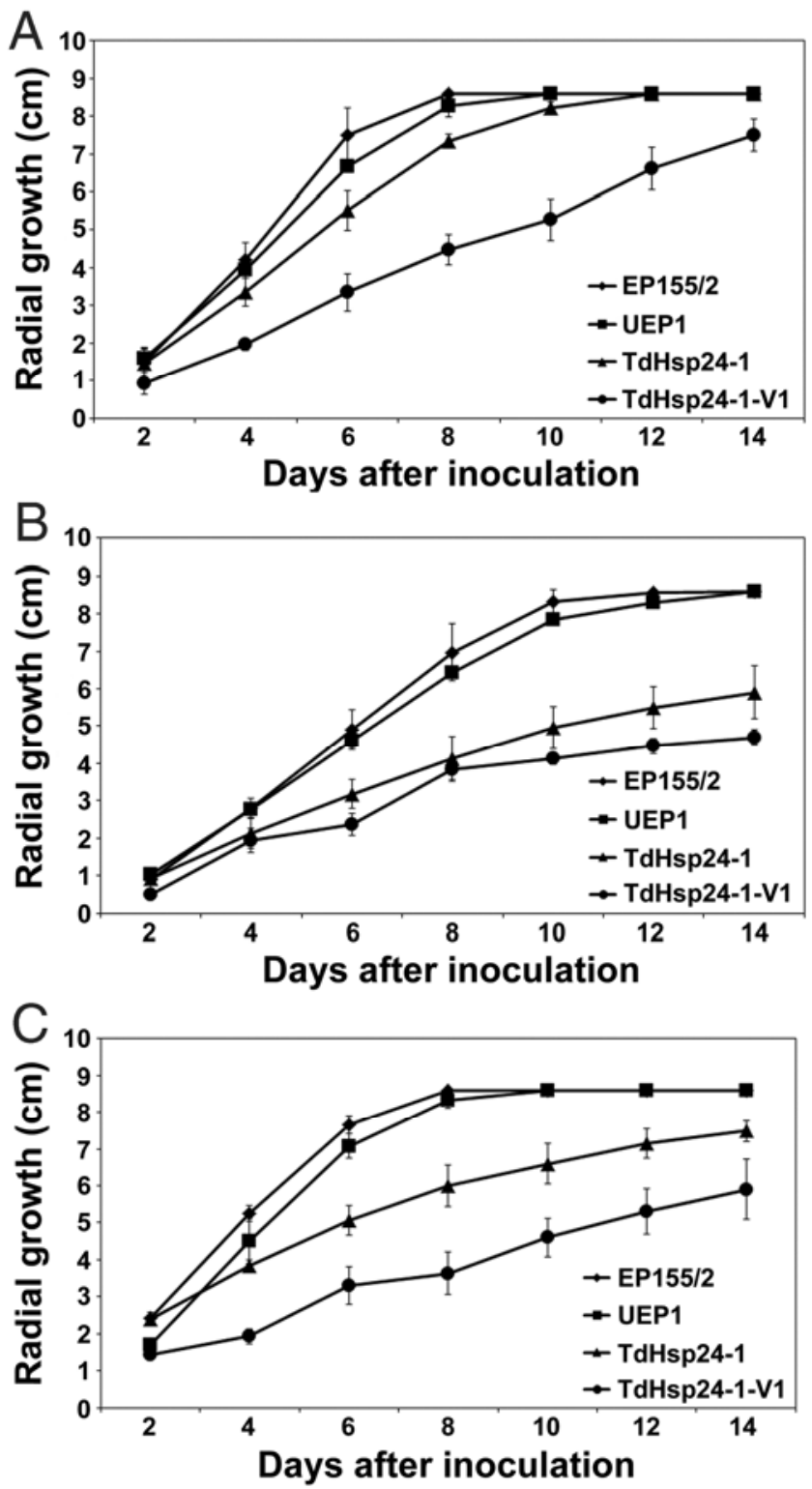

Fig. 4. Growth rate of the CpHsp24-null mutant strain under $\mathbf{A}$, standard temperature $\left(25^{\circ} \mathrm{C}\right)$ conditions; $\mathbf{B}$, cold shock $\left(20^{\circ} \mathrm{C}\right)$ conditions; and $\mathbf{C}$, heat-shock $\left(30^{\circ} \mathrm{C}\right)$ conditions. Strains used, indicated above the panel, are the wild-type EP155/2, hypovirulent UEP1, CpHsp24-null mutant (TdHsp24-1), and hypovirus-transferred CpHsp24-null mutant (TdHsp241-V1) strains. Vertical bars represent the standard deviation. growth defects due to cold shock $\left(20^{\circ} \mathrm{C}\right)$ than heat shock $\left(30^{\circ} \mathrm{C}\right)$. However, when the CpHsp24-null mutant strain was placed under other stresses such as oxidative and osmotic stresses, the responsiveness determined by the ratios between the growth rates in the presence or absence of menadione and high osmotic stress, respectively, were not changed. These results indicated that $\mathrm{CpHsp} 24$ contributed to adaptation to temperature stress but not to oxidative or osmotic stresses.

To ensure that the phenotypic changes attributed to the CpHsp24-null mutant were due to gene replacement of CpHsp24, we complemented the CpHsp24-null mutant in trans with a wild-type allele of the $C p H s p 24$ gene. The resulting geneticin-resistant transformants were compared with examined growth rate. No growth defects under standard growth conditions as well as heat-shock conditions were observed in the CpHsp24-complemented transformant (Fig. 3), which unequivocally confirmed that the phenotype changes in the CpHsp24-null mutant were due to the disruption of the CpHsp24 gene.

The oxidase activity of phenolic compounds by the strains measured by the browning of the plates containing TA was examined. Compared with the wild type, the CpHsp24-null mutant produced a brown-colored area with a size and intensity comparable with that of the wild type (Fig. 5). Considering the fact that the $\mathrm{CpHsp24-null} \mathrm{mutant} \mathrm{showed} \mathrm{reduced} \mathrm{growth}$ rates under standard growth conditions, no further reduction in the size and intensity of the browning area suggested that phenol oxidase production was not affected by the mutation of CpHsp24. Interestingly, even if the CpHsp24 gene was strongly induced by TA supplementation, no further changes in the growth rate were observed on TA-containing malt extract Bavendamm's media. However, when the CpHsp24-null mutant was placed on nutrient-poor minimal media supplemented with TA, further growth retardation ( $>10 \%$ more) was observed (Fig. 5), which might account for a significant induction of the CpHsp24 transcript by TA supplementation.

\section{Effect of hypoviral infection}

on the colony morphology of the CpHsp24-null mutant.

In order to examine the biological functions of the CpHsp24 gene related to hypovirus infection, we compared the pheno-

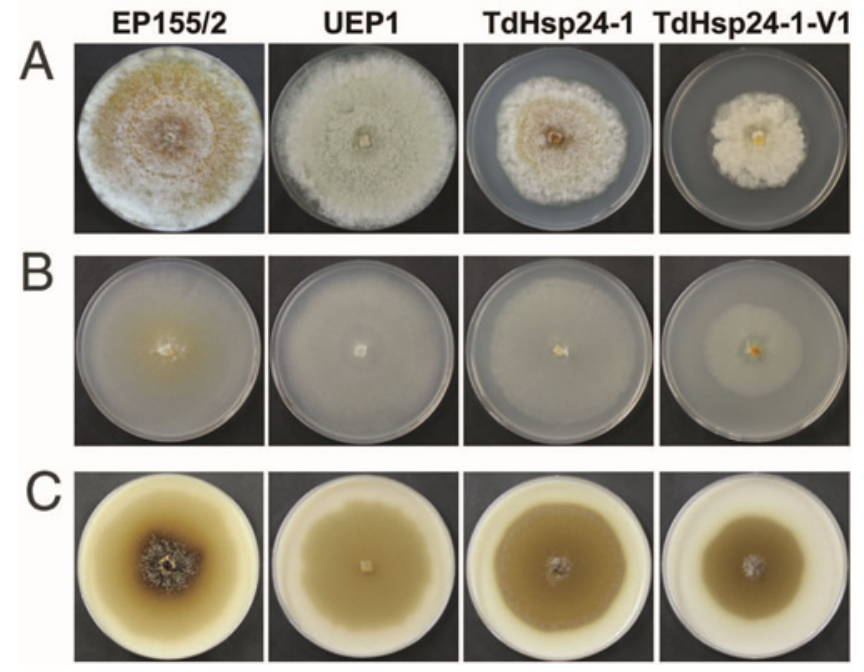

Fig. 5. Phenol oxidase activity of the $C p H s p 24$-null mutant strain. Colonies after 10 days of culturing on $\mathbf{A}$, potato dextrose agar supplemented with methionine and biotin; $\mathbf{B}$, malt extract media; and $\mathbf{C}$, malt extract media with tannic acid (TA) supplementation are shown. Strains used, indicated above the panel, are the wild-type EP155/2, hypovirulent UEP1, CpHsp24-null mutant (TdHsp24-1), and hypovirus-transferred CpHsp24null mutant (TdHsp24-1-V1) strains. 
typic changes between the virus-free and virus-containing isogenic transformants. Following co-culturing of the recipient CpHsp24-null mutant with UEP1, at least three putative viruscontaining recipient mycelia sections were independently transferred onto a new hygromycin B-containing PDAmb plate and single spored to ensure nuclear homogeneity on the hygromycin B-containing medium. The single-spored strains from different sections were checked for the presence of double-stranded (ds)RNA by agarose gel electrophoresis before further comparisons (data not shown). When the hypovirus CHV1-713 was transferred to the $C p H s p 24$-null mutant, colonial growth of the virus-containing $C p H s p 24$-null mutant was severely restricted compared with the virus-free CpHsp24-null mutant (Fig. 4). Further growth inhibition of the virus-containing $\mathrm{CpHsp24-null}$ mutant was observed at 20 and $30^{\circ} \mathrm{C}$, suggesting the sensitivity of the mutant to the temperature shock stress in the presence of the hypovirus (Fig. 4). In addition, reduced pigmentation and asexual sporulation, characteristic phenotypic changes caused

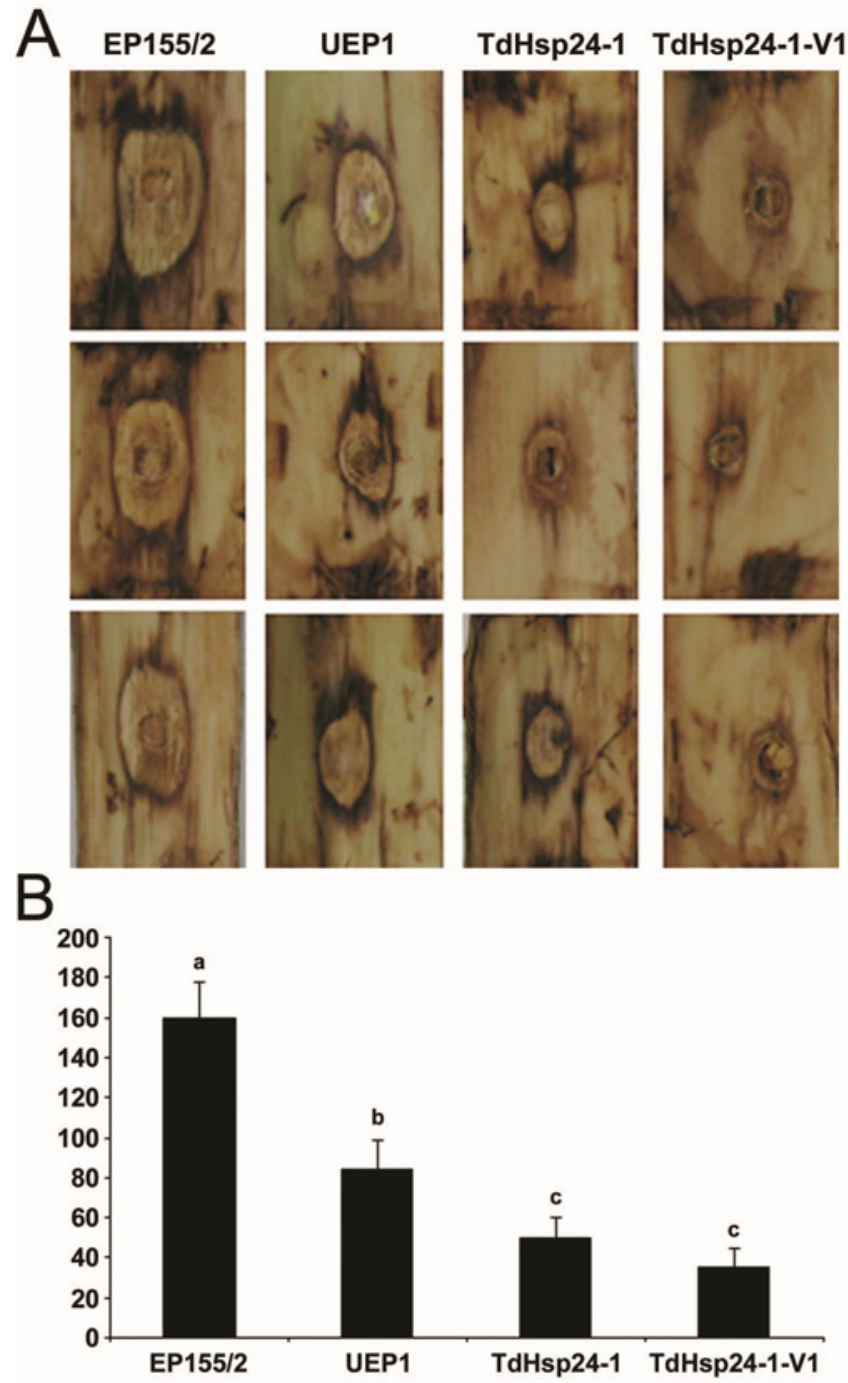

Fig. 6. Virulence assay using excised chestnut tree bark as previously described (Lee et al. 1992). The strains used were the wild-type EP155/2, hypovirulent UEP1, CpHsp24-null mutant (TdHsp24-1), and hypovirus-transferred CpHsp24-null mutant (TdHsp24-1-V1) strains. A, Representative figure of excised tree barks 1 week after inoculation. B, Lesion measurement values are shown as the means \pm standard deviation (square millimeters). Three replicates for each strain were used, and each experiment was repeated twice. Different letters indicate significant differences between treatments according to Duncan's multiple range test at $P=0.05$. by hypovirus infection, were observed in the virus-containing CpHsp24-null mutant (Fig. 5). These phenotypic characteristics of the virus-containing $C p H s p 24$-null mutant were stably maintained during the successive subculture of the virus-containing null mutant, which suggests that the presence of the CpHsp24 gene is required for the appropriate response of $C$. parasitica to hypoviral infection but is not required for the maintenance of the hypovirus.

\section{Virulence assay.}

A virulence assay on the excised bark from a chestnut tree demonstrated that the size of the necrotic area induced on excised bark by the CpHsp24-null mutant was significantly smaller than that induced by the wild-type EP155/2, indicating that the CpHsp24-null mutant is less virulent than the wild type. In addition, the size of the necrotic area of the CpHsp24null mutant was even smaller than that of a hypovirulent UEP1 strain (Fig. 6). Statistical analysis using multiple range test at $P=0.05$ indicated that the virulence of the CpHsp24-null mutant differed significantly from that of the wild type and even differed from that of the UEP1. Although it is known that pathogenicity tests are tightly correlated with the growth defects of the mutant, the remarkable reduction in the size of the necrotic area of the CpHsp24-null mutant suggested that the CpHsp24 gene is involved in determining the virulence of this fungus.

\section{Molecular characteristics of the CpHsp24-null mutant.}

Because the expression pattern of the $\mathrm{CpHsp24}$ gene (i.e., induction by TA supplementation that was hampered by hypovirus) was similar to that of the laccase 3 gene (lac3) gene, an important virulence factor of $C$. parasitica (Chung et al. 2008), and the virulence of the CpHsp24-null mutant was severely reduced, we examined lac3 expression in the CpHsp24-null mutant using quantitative real-time RT-PCR (Fig. 7). Interestingly, the accumulation of lac3 transcript in response to TA supplementation was hampered in the CpHsp24-null mutant. Therefore, it is suggested that the reduced lac 3 induction may at least partially account for the CpHsp24-null mutant's virulence attenuation. Although the similar expression pattern and the impaired virulence do not necessarily mean the interaction of two gene products, it would be of interest to examine whether the protein product of the CpHsp24 gene plays a part as a molecular chaperone for the LAC3 protein.

\section{DISCUSSION}

In order to understand fungal gene expression during pathogenic conditions, it is better to study the gene expression in the natural infection site; for example, the chestnut tree. However, in planta analyses of the pathogenesis of forest fungal pathogens have been considered to be more difficult than those of grass pathogens. In the case of chestnut blight by $C$. parasitica, supplementation with TA, which has been found in high concentrations in chestnut bark and is known to be a natural barrier against pathogen infection, was suggested as an alternative for mimicking host conditions. For example, the Bavendamm assay, which measures the degree of oxidizing activity on TA-supplemented plates, has been considered to be one of the best alternatives for measuring virulence (Rigling et al. 1989). Second, TA supplementation induced the expression of the lac 3 gene, which has been known to be a fungal virulence factor. Thus, it was believed that TA supplementation could create conditions more reflective of natural infection. The current study demonstrated that the specific Hsp gene CpHsp24 is influenced by TA supplementation and appears to be important for fungal virulence. This can be used as another example for validating the use of TA supplementation for analyzing viru- 
lence in this host-parasite system. Recent transcriptome and proteome analysis of mycelia grown under the presence of TA revealed that several genes are affected by TA and hypovirus infection (Kim et al. 2012). Thus, functional analysis of these affected genes could provide more information on the fungal response in the presence of natural host barriers.

The heat responsiveness of the accumulated transcript and protein products of the cloned $\mathrm{CpHsp24}$ gene indicated that the $C p H s p 24$ gene belongs to a member of Hsp. Moreover, the presence of a conserved region with the signature sequence of the $\alpha$-crystalline superfamily domain and the estimated molecular size of the protein product of the $C p H s p 24$ gene suggested that the cloned $C p H s p 24$ gene from $C$. parasitica encoded an sHsp that was a member of the Hsp23 family of molecular chaperones. In silico analysis of the promoter region $2.0 \mathrm{~kb}$ upstream of the start codon identified four nonconventional HSE motifs and two STRE motifs. These functional elements explain the heat-shock response and the TA induction of the CpHsp24 gene. However, not all types of stress induced the expression of the CpHsp24 gene and this induction was affected by hypovirus infection, suggesting the presence of a more complicated regulatory hierarchy in this promoter.

The biological function of sHsp in pathogenic microorganisms is largely unknown (Mayer et al. 2012). In yeast, multiple sHsp, including Hsp12, Hsp26, and Hsp30, exhibit different induction patterns in response to different stresses ( $\mathrm{Fu}$ et al. 2012). The requirements for growth and survival of these proteins under a variety of stress conditions as well as the requirements for the maintenance of normal cell morphology have been assessed (Welker et al. 2010). In addition to stress adaptation, the involvement of these Hsp in the virulence of eukaryotic pathogens (Mayer et al. 2012) and intracellular signaling in viral infections has been suggested (Rajaiya et al. 2012). In this study, Northern blot analysis demonstrated that the accumulation of the $\mathrm{CpHsp} 24$ gene transcript was significantly induced by TA supplementation and temperature stress. In addition, severe growth defects were observed when the CpHsp24null mutant was placed under stress conditions such as temperature and TA supplementation. These results clearly indicated that the $C p H s p 24$ gene is involved in stress adaptation. Although sHsp, unlike the high molecular mass Hsp, have been shown to be mainly expressed under stress conditions, Western blot analysis has indicated that the protein product of the CpHsp24 gene was found in considerable concentrations even in the absence of any stress, suggesting a physiological role under standard growth conditions. These results might explain the growth retardation of the CpHsp24-null mutant under standard growth conditions.

A severely reduced area of necrosis was observed on the excised bark of chestnut trees, which suggested that the CpHsp24 gene is involved in the pathogenicity of $C$. parasitica. Considering that pathogenicity tests of the loss-of-function mutant of the Hsp DnaJ, the first characterized Hsp gene of C. parasitica at the molecular level, appeared to be the mere reflection of the growth defect of the mutant (Lim et al. 2010), the dramatically reduced pathogenicity (even smaller than that of the hypovirulent UEP1) of the CpHsp24-null mutant appears to be specific. This is very interesting because only a few studies have described the role of sHsp in microbial pathogenicity; two bacterial (Stewart et al. 2005; Tsai et al. 2009) and one fungal (Mayer et al. 2012) pathogenicity. Based on the high induction pattern of the CpHsp24 gene by TA supplementation and the limited pathogenicity of the CpHsp24-null mutant, it

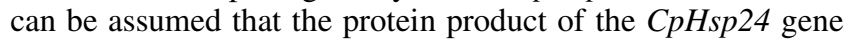
is implicated in chaperone activity of the virulence factors. sHSP have been shown to bind non-native proteins in an ATPindependent manner, maintaining them in a folding-competent state ready for refolding by other ATP-dependent components of protein quality control systems (Basha et al. 2012). Although some sHSP interact with a whole suite of denaturing proteins, many sHSP have specific substrates in vivo. Thus, further studies are needed to determine whether CpHsp24 is the major chaperone during the infection process or has specific protein partners involved in pathogenicity. It would be of great interest to clarify how the protein product of the $C p H s p 24$ gene functions in vivo and what the major in vivo substrates of the CpHSP24 protein are. Considering the similarities in the expression profiles of the lac 3 gene, an important virulence factor of $C$. parasitica (Chung et al. 2008), and the CpHsp24 gene under TA induction and hypovirus infection, as well as the abnormal expression of the lac3 gene in the CpHsp24-null mutant, it is tempting to speculate that $\mathrm{CpHsp24}$ might function as a molecular chaperone for the protein product of the lac3 gene. Therefore, further studies on the CpHsp24-lac3 interaction could provide information on the former's in vivo function as well as point the way for further specificity analysis of the $C$ pHsp24.

In addition to reduced pigmentation and sporulation, which are characteristic hypovirulence-associated phenotypic changes of the CHV1-infected hypovirulent strain, viral transfer to the CpHsp24-null mutant resulted in severe growth retardation compared with the virus-free $C p H s p 24$-null mutant. These results suggested that the presence of $\mathrm{CpHsp24}$ is required for the appropriate fungal response to a biotic stress such as hypovirus infection. This growth retardation was further increased when the virus-infected $C p H s p 24$-null mutant was cultured under temperature stress conditions. $C$. parasitica must adapt to a variety of stresses and it is likely that some of these stresses occur simultaneously. These results suggest that there appear to be two different stress-response pathways: one for abiotic stress such as temperature and another for biotic stress such as hypovirus infection. The presence of CpHsp24 is required for appropriate adaptation of the fungus to both abiotic and biotic stresses. Although the Hsp has been implicated as a molecular chaperone for viral replication and assembly (Sullivan and Pipas 2001), persistent growth retardation of the virus-infected $\mathrm{CpHsp24-null} \mathrm{mutant} \mathrm{without} \mathrm{reverting} \mathrm{pheno-}$ types, such as rapidly growing sectors shown in the virusinfected prol-null mutant (Sun et al. 2009), provided evidence of the stable maintenance of CHV1-EP713 in the CpHsp24-

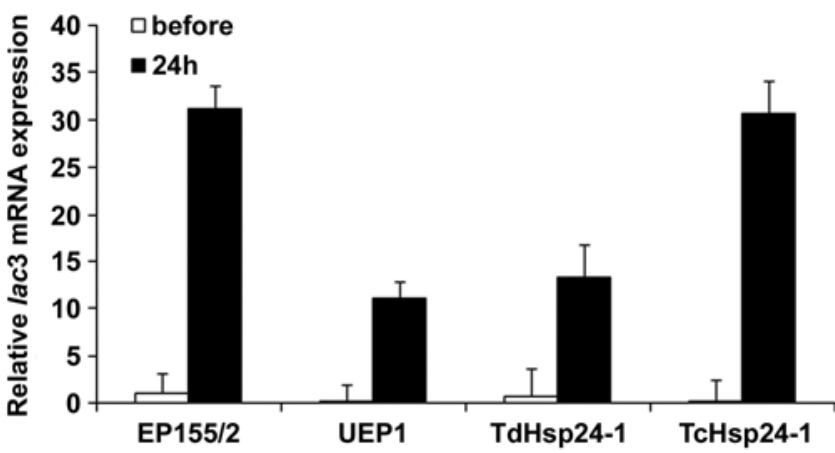

Fig. 7. Molecular changes in the accumulation of the lac 3 gene transcript in response to tannic acid (TA) supplementation. Total RNA was extracted before and $24 \mathrm{~h}$ after TA induction. The expression level of the CpHsp24null mutant (TdHsp24-1) was compared with the wild-type EP155/2, hypovirulent UEP1, and complemented CpHsp24-null mutant (TcHsp24-1) strains. Quantitative real-time reverse-transcription polymerase chain reaction analysis of lac3 transcript levels relative to the levels of $g p d$. Values on the $y$-axis were normalized to the transcript levels of the corresponding gene in the 5-day cultured EP155/2 strain, with standard deviations, based on three independent measurements of two independent RNA preparations, indicated by the error bars. 
null mutant and, thereby, indicated that the CpHsp24 gene may not be required for hypovirus replication. Bioassay data using the CpHsp24-null mutant suggested that the presence of CpHsp24 is important for fungal growth in hypovirus infections. Transcriptional analysis, however, revealed that the induction of $C p H s p 24$ by TA supplementation has been hampered by the hypovirus. Considering that the CpHsp24 gene is not required for the hypoviral replication, it is suggested that the presence of the $\mathrm{CpHsp24}$ gene is more beneficial to the fungal biology. Accordingly, the disturbance of CpHsp24 induction by the hypovirus may result in a fungal condition favoring viral persistence. Therefore, it would be of interest to examine the

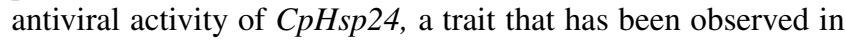
other sHsp (Arockiaraj et al. 2012; Rajaiya et al. 2012).

The current study represents the first detailed description of an sHsp in C. parasitica and shows an sHsp contributing to the stress adaptation and virulence of a eukaryotic pathogen. In addition, this study suggests the putative partner protein of the CpHSP24 chaperone, which could be an interesting model for studying the function of molecular chaperones during pathogenesis.

\section{MATERIALS AND METHODS}

\section{Fungal strains and growth.}

The CHV1-713 containing hypovirulent $C$. parasitica strain UEP1 and its isogenic virus-free strain EP155/2 (American Type Culture Collection 38755) were maintained on PDAmb plates under constant low light at $25^{\circ} \mathrm{C}$ (Kim et al. 1995). The culture conditions and methods for preparation of the primary inoculum for liquid cultures have been previously described (Kim et al. 1995). TA (Sigma-Aldrich, St Louis) was supplemented to the media as previously described (Rigling and Van Alfen 1993). TA induction of EP155/2 and UEP1 was performed according to Chung and associates (2008). Radial growth on plates was assessed by measuring the diameter of the colonies. The mycelium was collected and lyophilized as previously described until its use (Powell and Van Alfen 1987).

\section{RNA differential display analysis using the PCR-based GeneFishing technique.}

RNA differential display with the GeneFishing technique was conducted to examine the differential expression gene (DEG) between virus-free EP155/2 and hypovirulent UEP1 with or without TA supplementation. GeneFishing DEG kit (SeeGene Inc., Seoul, Korea) was used according to the manufacturer's instructions. Briefly, for first-strand synthesis, $2 \mu \mathrm{g}$ of DNA-free total RNA was converted into ssDNA using control primer one $(10 \mu \mathrm{M}$ dT-ACP1), two (dT-ACP2, which prime from polyA tail), dNTP (2 mM each), $2.5 \mu \mathrm{l}$ of $25 \mathrm{mM}$ $\mathrm{MgCl}_{2}, 2 \mu \mathrm{l}$ of RNase inhibitor (40 U/ $\mu \mathrm{l}$; Promega Corp.), and $1 \mu \mathrm{l}$ of reverse transcriptase (200 U/ $\mu \mathrm{l}$; Promega Corp.). The volume of the reaction mixture was $20 \mu \mathrm{l}$, and it was allowed to react for $60 \mathrm{~min}$ at $42^{\circ} \mathrm{C}$ and then for $15 \mathrm{~min}$ at $70^{\circ} \mathrm{C}$. For second-strand synthesis, the PCR was performed as one cycle at $94^{\circ} \mathrm{C}$ for $3 \mathrm{~min}$, followed by $50^{\circ} \mathrm{C}$ for $3 \mathrm{~min}$ and $72^{\circ} \mathrm{C}$ for $1 \mathrm{~min}$. After the ssDNA synthesis was completed, the secondstage PCR amplification protocol was 40 cycles at $94^{\circ} \mathrm{C}$ for $40 \mathrm{~s}, 65^{\circ} \mathrm{C}$ for $40 \mathrm{~s}$, and $72^{\circ} \mathrm{C}$ for $40 \mathrm{~s}$; and then a 5 -min final extension step at $72^{\circ} \mathrm{C}$. In total, 20 different annealing control primer (ACP) pairs were used (each primer's sequence can be found in the SeeGene Inc. protocol). ACP primers were designed for highly specific PCR in second-stage PCR amplification. The differentially expressed bands were cut out of the gel using a QIAquick gel extraction kit (Qiagen, Carlsbad, CA, U.S.A.), directly cloned into pGEM-T Easy vectors (Promega Corp.), and then sequenced.
GeneFishing DEG analysis resulted in a cloned 401-bp PCR amplicon exhibiting a high homology with known fungal small heat-shock genes (Lindquist and Craig 1988; Plesofsky 2004). A genome database of $C$. parasitica (Cryphonectria parasitica EP155 v2.0) was screened for a full-length gene, and PCR amplification was performed with the primers CpHsp24-gF1 (forward) 5'-TTCTCAAGCCACCCAAAGTCA-3' and CpHsp24-gR1 (reverse) 5'-CGTACGTTCACATGCGCGTA T-3'. The resulting 4.0-kb PCR amplicon was cloned into the pGEM-T Easy Vector (Promega Corp.) and sequenced using the dideoxynucleotide method with universal and synthetic oligonucleotide primers.

In order to obtain the cDNA clone of CpHsp24, RT-PCR

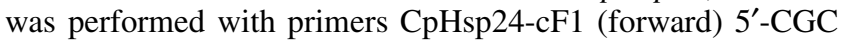
GGA TCC ATG GCT GAA GAC ATC ATC CAT-3' and CpHsp24-cR1 (reverse) 5'-ACG CGT CGA CTC AGT GGC CCG CAT GTT CGA T-3'. The primers were modified to incorporate the restriction sites (underlined) for BamHI and SalI, respectively. The cDNA was sequenced using the dideoxynucleotide method with synthetic oligonucleotide primers.

\section{Southern blot and Northern blot analysis.}

Genomic DNA from $C$. parasitica was extracted using the method described by Churchill and associates (1990). DNA $(10 \mu \mathrm{g})$ was digested with restriction enzymes BamHI and SacI, blotted onto the nylon membrane, and hybridized with radioactive-labeled probes.

RNA from liquid cultures was extracted as previously described (Kim et al. 2002). RNA from solid cultures was prepared from mycelia mats grown on cellophane that was layered on top of appropriate media, as previously described (Park et al. 2004). The level of CpHsp24 transcript was compared against glyceraldehyde-3-phosphate dehydrogenase $(G p d)$ of C. parasitica as an internal control for gene expression (Chio and Nuss 1990).

\section{Heterologous expression of CpHsp24 in Escherichia coli.}

The full-length $\mathrm{CpHsp24}$ protein product CpHSP24 was expressed in Escherichia coli as a hexahistidine fusion protein and purified by nickel affinity chromatography according to the manufacturer's directions (Qiagen). The full-length open reading frame of CpHsp24 (648 bp) was inserted into the BamHI/SalI sites in the expression vector pQE30 (Qiagen). The resulting recombinant plasmids were transformed into $E$. coli strain M15, including the pREP4 repressor vector. The induction, purification, and confirmation of the recombinant CpHSP24 using the anti-hexahistidine antibody were conducted according to the manufacturer's directions (Qiagen). The E. coli-derived inclusion body consisting of the recombinant CpHSP24 was solubilized and then refolded by the stepwise dilution dialysis of denaturants (Sambrook and Russell 2001).

The anti-CpHSP24 antibody was obtained by injecting 100 $\mu \mathrm{g}$ of purified full-length $\mathrm{CpHSP} 24$ into an 8-week-old BALB/c mouse, which was boosted with the same amount of the CpHSP24 emulsified in incomplete Freund's adjuvant 10 days after the initial injection. Polysera were obtained 5 days after the booster injection and then Western blot analysis was conducted according to the standard procedure (Sambrook and Russell 2001).

\section{Construction of a replacement vector and fungal transformation.}

The replacement vector pDHsp24, which was designed to favor double-crossover integration events, was constructed as follows. A 4,047-bp NotI fragment containing the full-length CpHsp24 was ligated into SalI-inactivated pBluescriptII SK 
$(+)$, and the resulting plasmid was used as the template for inverted PCR using the primers $5^{\prime}$-ACGCGTCGACATCCAC AATACGCGCGCAC-3' $3^{\prime}$ and $5^{\prime}$-ACGCGTCGACCTTGTTGT GTGCGGGTGCG-3', which incorporate the restriction site for SalI (underlined). The PCR amplicon was digested with SalI and religated. The resulting plasmid was further digested with SalI and fused with a 2.4-kb SalI fragment of pDH25 (Cullen et al. 1987) carrying the $h p h$ gene cassette. In the replacement vector pDHsp24, the $h p h$ cassette was inserted between sites 1 and 648 of the CpHsp24 gene relative to the start codon, and was flanked by approximately 1,176 and $1,355 \mathrm{bp}$ of the $5^{\prime}$ and $3^{\prime}$ sequences, respectively. A 4,398-bp PCR fragment containing the $h p h$ cassette and flanking regions of the CpHsp24 gene was obtained from pDHsp24 with the primers CpHsp24-gF1 and CpHsp24-gR1, and was then used to transform the virusfree EP155/2 strain.

Functional complementation of the CpHsp24 null mutant using a wild-type allele was performed. The complementing vector pCHsp24 was constructed by the insertion of a $1.7-\mathrm{kb}$ blunt-ended XhoI fragment of pSilent-Dual1G (pSD1G) containing the geneticin resistance cassette (Nguyen et al. 2008) into SacII/SpeI-digested pCHsp24 carrying the 4,047-bp fragment with the full-length $C p H s p 24$ gene. The resulting vector was then used to transform the CpHsp24 null mutant.

Protoplast preparation and transformation were performed as previously described (Churchill et al. 1990; Kim et al. 1995). Transformants were selected from agar plates that were supplemented with hygromycin B (Calbiochem, San Diego, CA, U.S.A.) at $150 \mu \mathrm{g} / \mathrm{ml}$ or geneticin (Invitrogen, Carlsbad, CA, U.S.A.) at $150 \mu \mathrm{g} / \mathrm{ml}$, as was appropriate; passaged three to four times on the selective media; and single-spore isolated, as previously described (Kim et al. 2004; Nguyen et al. 2008). PCR and Southern blot analysis was conducted with genomic DNA from the transformants to confirm the replacement and in trans complementation of the CpHsp24 gene.

\section{Characteristics of the CpHsp24 null mutant.}

The phenotypic and molecular characteristics of the CpHsp24 null mutant were compared with those of the wildtype EP155/2 and the hypovirulent UEP1 strains. Phenotypic changes in pigmentation and conidiation were measured as previously described (Kim et al. 2002; Powell et al. 1987). A virulence test using excised chestnut tree bark was conducted according to Lee and associates (1992). Phenol oxidase activity was gauged by growing the strains on Bavendamm's medium (0.05\% TA, $1.5 \%$ malt extract, and $2.0 \%$ agar) and assessing the resulting coloration of the medium (Rigling et al. 1989).

To examine the acute wet heat-shock response, the actively growing $C$. parasitica mycelia on cellophane that was layered on top of PDAmb medium were ground, transferred to Endothia parasitica (EP) complete media, and incubated at $25^{\circ} \mathrm{C}$ for 3 days. The resulting mycelia were harvested, dried using paper towels until no further liquid leaked out, transferred to EP complete medium preequilibrated to appropriate temperatures, and, finally, incubated further for $24 \mathrm{~h}$. The assessment of the response to chronic temperature stress was based on the level of hyphal growth on PDAmb incubated at appropriate temperatures. Northern blot and Western blot analyses were conducted to examine the accumulation of the transcript and protein product of the $C p H s p 24$ gene, respectively.

\section{Transmission of dsRNA virus.}

Virus transmission was performed as previously described (Kim et al. 2008). Briefly, mycelial plugs of the virus-containing strain UEP1 were co-cultured with adjacent mycelial plugs of virus-free recipient transformants on PDAmb medium. After 5 days of co-culture, putatively fused mycelia at the border be- tween each pair of strains were transferred to hygromycincontaining PDAmb and examined for the occurrence of a sector that showed different colonial phenotypes, such as reduced growth or pigmentation. Mycelia in the sector were successively transferred to fresh hygromycin-containing media and then strains were single spored to select for the virus-infected recipient transformants. The presence of hypovirus was confirmed by purification of dsRNA from the single-spored isolates.

\section{Isolation of dsRNA from $C$. parasitica.}

The dsRNA was isolated according to the modified procedure of Kim and associates (2008). The UEP1 and dsRNAtransmitted transformants were grown on a cellophane membrane overlaying PDAmb for 5 days at $25^{\circ} \mathrm{C}$. Mycelia $(0.2 \mathrm{~g}$ wet weight) were homogenized in extraction buffer $(2 \times$ STE [0.2 M NaCl, 0.1 M Tris-HCl [pH 8.0], and $2 \mathrm{mM}$ EDTA], 2\% SDS, and $1 \%$ sodium bisulfate) using a bead beater (Biospec Products, Inc., Bartlesville, OK, U.S.A.). Following two successive phenol extractions, dsRNA was isolated by affinity chromatography using CC41 resin. Isolated dsRNA was analyzed by electrophoresis in a $0.8 \%$ agarose gel.

\section{Quantitative analysis of lac3 transcript accumulation using real-time RT-PCR.}

To examine the expression levels of target and internal control genes, quantitative RT-PCR was performed using a GeneAmp 7500 sequence detection system (Applied Biosystems, Foster City, CA, U.S.A.) and a SYBR green mixture RT kit (Applied Biosystems). A single cycle to reverse-transcribe $1 \mu \mathrm{g}$ of RQ1-treated RNA was followed by real-time PCR using $2 \mu \mathrm{l}$ of reverse-transcribed cDNA and $150 \mathrm{nM}$ each of forward and reverse primers. Analyses were conducted at least twice in triplicate for each transcript and were from at least two independent RNA preparations with primers specific for gpd and the target genes. The $g p d$ was amplified with the primers RTgpd-F (5'-CTCTCAACACGGCAACTTCA-3') and RT-gpd-R (5'-ACCAGTGGACTCGACAATG-3'). The lac3 transcript was amplified with the primers RT-lac3-F (5'-CACGGAATCCGA CAAAACTTC-3') and RT-lac3-R (5'-CTGCACGTAGAAGT GCGAGT-3'). Transcript abundance relative to the amount of gpd in the sample was calculated using the comparative threshold cycle method, as previously described (Parsley et al. 2002).

\section{Statistical analysis of virulence data.}

Necrotic areas of the virulence test were analyzed by analysis of variance using SPSS (version 15; SPSS, Chicago), with significant differences between strains determined by the magnitude of the $F$ value at $P=0.05$. When a significant $F$ value was obtained, the significance of the effects of the strains was determined by Duncan's multiple range test at $P=0.05$.

\section{ACKNOWLEDGMENTS}

This work was supported by the National Research Foundation of Korea grant funded by the Korea government (MSIP number 2008-0061897) and, in part, by the Bio-industry Technology Development Program, Ministry for Food, Agriculture, Forestry and Fisheries, Republic of Korea. This research was also supported, in part, by the Korea Research Council of Fundamental Science and Technology (Joint Degree and Research Center for Biorefinery). We thank the DOE Joint Genome Institute for the genome database of C. parasitica and Center for Fungal Pathogenesis and the Institute of Molecular Biology and Genetics at Chonbuk National University for kindly providing the facilities for this research.

\section{LITERATURE CITED}

Allen, T. D., and Nuss, D. L. 2004. Specific and common alterations in host gene transcript accumulation following infection of the chestnut blight fungus by mild and severe hypoviruses. J. Virol. 78:4145-4155. 
Allen, T. D., Dawe, A. L., and Nuss, D. L. 2003. Use of cDNA microarrays to monitor transcriptional responses of the chestnut blight fungus Cryphonectria parasitica to infection by virulence attenuating hypoviruses. Eukaryot. Cell 2:1253-1265.

Anagnostakis, S. L. 1982. Biological control of chestnut blight. Science 215:466-471.

Arockiaraj, J., Vanaraja, P., Easwvaran, S., Singh, A., Othman, R. Y., and Bhassu, S. 2012. Gene expression and functional studies of small heat shock protein 37 (MrHSP37) from Macrobrachium rosenbergii challenged with infectious hypodermal and hematopoietic necrosis virus (IHHNV). Mol. Biol. Rep. 39:6671-6682.

Basha, E., O'Neill, H., and Vierling, E. 2012. Small heat shock proteins and alpha-crystallines: dynamic proteins with flexible functions. Trends Biochem. Sci. 37:106-117.

Buchner, J. 1996. Supervising the fold: functional principles of molecular chaperones. FASEB (Fed. Am. Soc. Exp. Biol.) J. 10:10-19.

Choi, G. H., and Nuss, D. L. 1990. Nucleotide sequence of the glyceraldehyde-3-phosphate dehydrogenase gene from Cryphonectria parasitica. Nucleic Acid Res. 18:5566.

Chung, H. J., Kwon, B. R., Kim, J. M., Park, S. M., Park, J. K., Cha, B. J., Yang, M. S., and Kim, D. H. 2008. A tannic acid-inducible and hypoviral-regulated Laccase 3 contributes to the virulence of the chestnut blight fungus Cryphonectria parasitica. Mol. Plant-Microbe Interact. 21:1582-1590.

Churchill, A. C. L., Ciufetti, L. M., Hansen, D. R., Van Etten, H. D., and Van Alfen, N. K. 1990. Transformation of the fungal pathogen Cryphonectria parasitica with a variety of heterologous plasmids. Curr. Genet. 17:25-31.

Cullen, D., Leong, S. A., Wilson, L. J., and Henner, D. J. 1987. Transformation of Aspergillus nidulans with the hygromycin-resistance gene, hph. Gene 57:21-26.

de Jong, W. W., Leunissen, J. A., and Voorter, C. E. 1993. Evolution of the alpha-crystalline/small heat-shock protein family. Mol. Biol. Evol. 10:103-126.

Deng, F., Allen, T. D., Hillman, B. I., and Nuss, D. L. 2007. Comparative analysis of alterations in host phenotype and transcript accumulation following hypovirus and mycoreovirus infections of the chestnut blight fungus Cryphonectria parasitica. Eukaryot. Cell 6:1286-1298.

Elliston, J. E. 1985. Characteristics of dsRNA-free and dsRNA-containing strains of Endothia parasitica in relation to hypovirulence. Phytopathology 75:151-158.

Fu, M. S., de Sordi, L., and Mühlschlegel, F. A. 2012. Functional characterization of the small heat shock protein Hsp12p from Candida albicans. PLoS One 7:e42894. Published online.

Garstel, M., Vajdy, E., and Buchner, J. 1997. The small Hsps-An overview. Pages 269-272 in: Guidebook to the Molecular Chaperones and Protein-Folding Catalysts. Oxford University Press, Oxford.

Havir, E. A., and Anagnostakis, S. L. 1983. Oxalate production by virulent but not by hypovirulent strains of Endothia parasitica. Physiol. Plant Pathol. 23:369-376.

Henikoff, S., and Henikoff, J. G. 1992. Amino acid substitution matrices from protein blocks. Proc. Natl. Acad. Sci. U.S.A. 89:10915-10919.

Jaya, N., Garcia, V., and Vierling, E. 2009. Substrate binding site flexibility of the small heat shock protein molecular chaperones. Proc. Natl. Acad. Sci. U.S.A. 106:15604-15609.

Ju, Y. J., Kim, D. H., and Cha, B. J. 2002. Isolation of Cryphonectria parasitica from cankers on chestnut trees in Korea. Plant Pathol. J. 18:23-29.

Kang, H. S., Choi, J. W., Park, S. M., Cha, B. J., Yang, M. S., and Kim, D. H. 1999. Ordered differential display from Cryphonectria parasitica. J. Plant Pathol. 16:142-146.

Kazmierczak, P., Pfeiffer, P., Zhang, L., and Van Alfen, N. K. 1996. Transcriptional repression of specific host genes by the mycovirus Cryphonectria hypovirus CHV1. J. Virol. 70:1137-1142.

Kazmierczak, P., McCabe, P., Turina, M., Jacob-Wilk, D., and Van Alfen, N. K. 2012. The mycovirus CHV1 disrupts secretion of a developmentally regulated protein in Cryphonectria parasitica. J. Virol. 86:60676074

Kim, D. H., Rigling, D., Zhang, L., and Van Alfen, N. K. 1995. A new extracellular laccase of Cryphonectria parasitica is revealed by deletion of Lac1. Mol. Plant-Microbe Interact. 8:259-266.

Kim, J. M., Park, J. A., and Kim, D. H. 2012. Comparative proteomic analysis of chestnut blight fungus, Cryphonectria parasitica, under tannic-acid-inducing and hypovirus-regulating conditions. Can. J. Microbiol. 58:863-871.

Kim, M. J., Choi, J. W., Park, S. M., Cha, B. J., Yang, M. S., and Kim, D. H. 2002. Characterization of a fungal protein kinase from Cryphonectria parasitica and its transcriptional upregulation by hypovirus. Mol. Microbiol. 45:933-941.

Kim, M. J., Park, S. M., Kim, Y. H., Cha, B. J., Yang, M. S., and Kim, D. H. 2004. Deletion of a hypoviral-regulated cppk1 gene in a chestnut blight fungus, Cryphonectria parasitica, results in microcolonies. Fungal Genet. Biol. 41, 482-492.

Kim, M. J., Kwon, B. R. Park, S. M., Chung, H. J., Yang, M. S., Churchill, A. C. L., Van Alfen, N. K., and Kim, D. H. 2008. Promoter analysis of the cell surface-abundant and hypoviral-regulated cryparin gene from Cryphonectria parasitica. Mol. Cells 26:496-502.

Lee, J. K., Tattar, T. A. Berman, P. M., and Mount, M. S. 1992. A rapid method for testing the virulence of Cryphonectria parasitica using excised bark and wood of American chestnut. Phytopathology 82:14541456.

Lee, J. K., Lee, H. Y. Lee, S. H., and Hwang, M. S. 1999. Assessment of tree susceptibility to chestnut blight fungus (Cryphonectria parasitica) of various chestnut varieties. Page D-45 in: (Abstr.) Annual Meeting of the Korean Society of Plant Pathologists, Suwon, Korea.

Lim, J. G., Lee, J. G., Kim, J. M., Park, J. A., Park, S. M., Yang, M. S., and Kim, D. H. 2010. A DnaJ-like homolog from Cryphonectria parasitica is not responsive to hypoviral infection but is important for fungal growth in both wild-type and hypovirulent strains. Mol. Cells 30:235243.

Lindquist, S., and Craig, E. A. 1988. The heat-shock proteins. Annu. Rev. Genet. 22:631-677.

Martínez-Pastor, M. T., Marchler, G., Schüller, C., Marchler-Bauer, A., Ruis, H., and Estruch, F. 1996. The Saccharomyces cerevisiae zinc finger proteins $M s n 2 p$ and Msn4p are required for transcriptional induction through the stress response element (STRE). EMBO (Eur. Mol. Biol. Organ.) J. 15:2227-2235.

Mayer, F. L., Wilson, D., Jacobsen, I. D., Miramón, P., Slesiona, S., Bohovych, I. M., Brown, A. J., and Hube, B. 2012. Small but crucial: the novel small heat shock protein Hsp21 mediates stress adaptation and virulence in Candida albicans. PLoS One 7:e38584. Published online.

Narberhaus, F. 2002. Alpha-crystallin-type heat shock proteins: socializing minichaperones in the context of a multichaperone network. Microbiol. Mol. Biol. Rev. 66:64-93.

Nguyen, Q. B., Kadotani, N., Kasahara, S., Tosa, Y., Mayama, S., and Nakayashiki, H. 2008. Systematic functional analysis of calcium-signalling proteins in the genome of the rice-blast fungus, Magnaporthe oryzae, using a high-throughput RNA-silencing system. J. Mol. Microbiol. 68:1348-1365.

Nuss, D. L. 1992. Biological control of chestnut blight: an example of virus-mediated attenuation of fungal pathogenesis. Microbiol. Rev. 56:561-576.

Park, S. M., Choi, E. S., Kim, M. J., Cha, B. J., Yang, M. S., and Kim, D. H. 2004. Characterization of HOG1 homologue, CpMK1, from Cryphonectria parasitica and evidence for hypovirus-mediated perturbation of its phosphorylation in response to hypertonic stress. Mol. Microbiol. 51:1267-1277.

Parsley, T. B., Chen, B., Geletka, L. M., and Nuss, D. L. 2002. Differential modulation of cellular signaling pathways by mild and severe hypovirus strains. Eukaryot. Cell 1:401-413.

Plesofsky, N. 2004. Heat shock proteins and the stress response. Pages 143-173 in: The Mycota III Biochemistry and Molecular Biology, 2nd ed. R. Brambl and G. A. Marzluf, eds. Springer-Verlag, Berlin.

Powell, W. A. J., and Van Alfen, N. K. 1987. Two nonhomologus viruses of Cryphonectria (Endothia) parasitica reduce accumulation of specific virulence-associated polypeptides. J. Bacteriol. 169:5324-5326.

Rajaiya, J., Yousuf, M. A., Singh, G., Stanish, H., and Chodosh, J. 2012 Heat shock protein 27 mediated signaling in viral infection. Biochemistry 51:5695-5702.

Richter, K., Haslbeck, M., and Buchner, J. 2010. The heat shock response: life on the verge of death. Mol. Cell 40:253-266.

Rigling, D., and Van Alfen, N. K. 1993. Intra- and extracellular laccases of the plant pathogenic fungus Cryphonectria parasitica. Appl. Environ. Microbiol. 59:3634-3639.

Rigling, D., Heiniger, U., and Hohl, H. R. 1989. Reduction of laccase activity in dsRNA-containing hypovirulent strains of Cryphonectria (Endothia) parasitica. Phytopathology 79:219-223.

Sambrook, J. S., and Russell, D. 2001. Molecular Cloning: A Laboratory Manual, 3rd ed. Cold Spring Harbor Laboratory Press, Cold Spring Harbor, NY, U.S.A.

Stewart, G. R., Newton, S. M., Wilkinson, K. A., Humphreys, I. R., Murphy, H. N., Robertson, B. D., Wilkinson, R. J., and Young, D. B. 2005. The stress-responsive chaperone alpha-crystallin 2 is required for pathogenesis of Mycobacterium tuberculosis. Mol. Microbiol. 55:1127-1137.

Sullivan C. S., and Pipas, J. M. 2001. The virus-chaperone connection. Virology 287:1-8.

Sun, Q., Choi, G., and Nuss, D. L. 2009. A silencing argonaute gene is required for induction of RNA silencing antiviral defense and promotes viral RNA recombination. Proc. Natl. Acad. Sci. U.S.A. 106:1792717932 . 
Tachibana T., Astumi, S., Shioda, R., Ueno, M., Uritani, M., and Ushimaru, T. 2002. A novel non-conventional heat shock element regulates expression of MDJ1 encoding a DnaJ homolog in Saccharomyces cerevisiae. J. Biol. Chem. 277:22140-22146.

Tsai, Y. L., Wang, M. H., Gao, C., Klusener, S., and Baron, C. 2009. Small heat-shock protein HspL is induced by VirB protein(s) and promotes VirB/D4-mediated DNA transfer in Agrobacterium tumefaciens. Microbiology 155:3270-3280.

Van Alfen, N. K. 1982. Biology and potential for disease control of hypovirulence of Endothia parasitica. Annu. Rev. Phytopathol. 20:349-362.

Van Alfen, N. K., Jaynes, R. A., Anagnostakis, S. L., and Day, P. R. 1975. Chestnut blight: biological control by transmissible hypovirulence in Endothia parasitica. Science 189:890-891.

Walter, S., and Buchner, J. 2002. Molecular chaperones-cellular machines for protein folding. Angew. Chem. Int. Ed. Engl. 41:1098-1113.
Wang, J., Wang, F., Feng, Y., Mi, K., Chen, Q., Shang, J., and Chen, B. 2013. Comparative vesicle proteomics reveals selective regulation of protein expression in chestnut blight fungus by a hypovirus. J. Proteomics 14:221-230.

Welker, S., Rudolph, B., Frenzel, E., Hagn, F., Liebisch, G., Schmitz, G., Scheuring, J., Kerth, A., Blume, A., Weinkauf, S., Haslbeck, M., Kessler, H., and Buchner, J. 2010. Hsp12 is an intrinsically unstructured stress protein that folds upon membrane association and modulates membrane function. Mol. Cell 39:507-520

\section{AUTHOR-RECOMMENDED INTERNET RESOURCE}

Department of Energy Joint Genome Institute draft genome sequence: genome.jgi-psf.org/Crypa1/Crypa1.home.html 